\title{
Heartbeat: Fixed dose combination drugs for hypertension
}

Fixed dose combination (FDC) drugs are standard practice in the treatment of infectious diseases, but their role in chronic non-communicable diseases is unclear. FDC anti-hypertensive drugs are an attractive option to improve compliance by reducing the number of pills taken daily, particularly in elderly patients who generally require more than one drug to control blood pressure and often are on multiple other medications. However, there are concerns that FDC antihypertensives might increase the risk of medication errors, specifically therapeutic duplications resulting in potentially serious drugdrug interactions, compared with prescribing each component of the FDC separately.

In order to answer this question, Moriarty and colleagues ${ }^{1}$ retrospectively evaluated all primary healthcare prescriptions in Ireland for the 2015 year, using the Primary Care Reimbursement Services administrative pharmacy claims database. The primary outcome was therapeutic duplication, defined as the use of an anti-hypertensive drug of the same class in an FDC preparation and as a separate drug, prescribed at the same time. The secondary outcome was the presence of a potentially serious drugdrug interaction involving a component of an included antihypertensive FDC and another co-prescribed medication. From 307,833 FDC prescriptions and 151632 separate component prescriptions for anti-hypertensive agents, therapeutic duplication was twice as likely with a FDC but still occurred in only $0.8 \%$ of patients, most often involving calcium channel blockers. The risk of a drug-drug interaction between medications prescribed a the same time (most often amlodipine and simvastatin) was about $10 \%$ but was no different for FDC compared with single component prescriptions.

The take-home message is that healthcare professionals or patients who use FDC anti-hypertensive medication

\footnotetext{
Internal Medicine, Universidade Federal de Minas Gerais, Belo Horizonte, Brazil

${ }^{2}$ Division of Cardiology, University of Washington, Seattle, Washington, USA
}

Correspondence to Professor Catherine M Otto, Division of Cardiology, University of Washington, Seattle,WA 98195, USA; cmotto@uw.edu

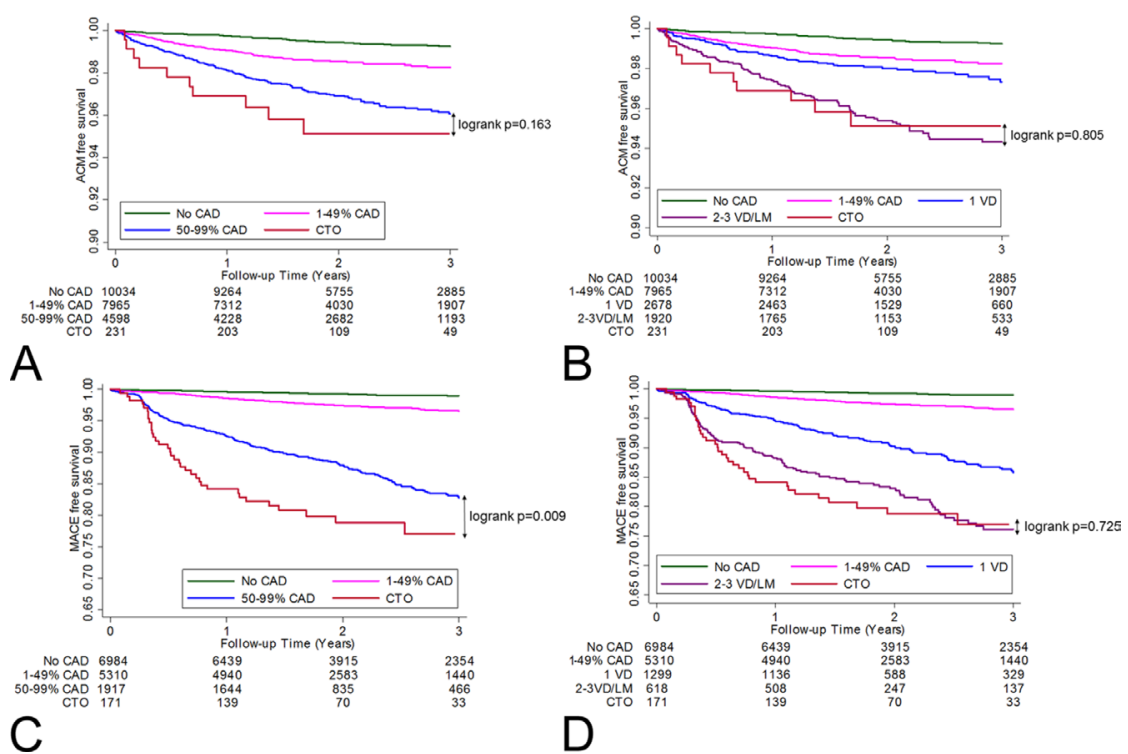

Figure 1 (A) All cause mortality (ACM)-free survival according to the severity of CAD and the presence or absence of CTO on CCTA. (B) ACM-free survival according to the extent of CAD and the presence or absence of CTO on CCTA. (C) Composite outcome of ACM, non-fatal myocardial infarction and late revascularisation-free survival according to the severity of $C A D$ and the presence or absence of CTO on CCTA. (D) Composite outcome of ACM, non-fatal myocardial infarction and late revascularisation-free survival according to the extent of $C A D$ and the presence or absence of CTO on CCTA. For the purpose of the per-vessel analysis, patients with one VD and multivessel or LM CAD had non-occlusive CAD. ACM, all-ause mortality; CAD, coronary artery disease; CCTA, coronary CT angiography; CTO, chronic total occlusion; LM, left main; MACE, major adverse cardiac events; $V D$, vessel disease.

should exercise caution and vigilance with these medications in order to minimise medication errors and patient harm. In the companion editorial, Webster ${ }^{2}$ considered these findings reassuring for high incomes settings, where FDCs are broadly available, considering the low duplication rates and absence of a higher risk of serious drug-drug interactions. She emphasises also that FDCs for chronic non-communicable diseases have a great potential to improve cardiovascular risk factors in low- and middle-income countries, where most of burden of chronic non-communicable diseases lies and where the availability of FDCs currently is limited.

Although the presence of a coronary artery chronic total occlusion (CTO) is associated with a higher rate of adverse cardiac events in patients after myocardial infarction or with stable coronary artery disease (CAD), the significance of a CTO identified on coronary computed tomographic angiography (CCTA) in patents with no prior history of CAD is unclear. In a registry of over 22 thousand patients without known CAD with follow-up data for a median of 26 months, a CTO was present in only $1 \%$ of patients. ${ }^{3}$ The mortality rate for CTO patients was no different than the $20 \%$ of patients with moderate-severe CAD (22.95; $95 \%$ CI 12.71 to 41.45 vs 14.46 ; $95 \%$ CI 12.34 to $16.94 ; \mathrm{P}=0.163$ ) but was higher than patients with nonobstructive CAD or normal coronary arteries. However, patients with a CTO had higher event rates than those with moderate-severe CAD, mainly due to a higher rate of later revascularisation (figure 1).

In the accompanying editorial, Azevedo and Bittencourt ${ }^{4}$ emphasise that patients referred for a CCTA for suspected CAD who are found to have a CTO have a different risk profile and prognosis compared with symptomatic patients with a CTO in the setting of known coronary disease or prior 
Women

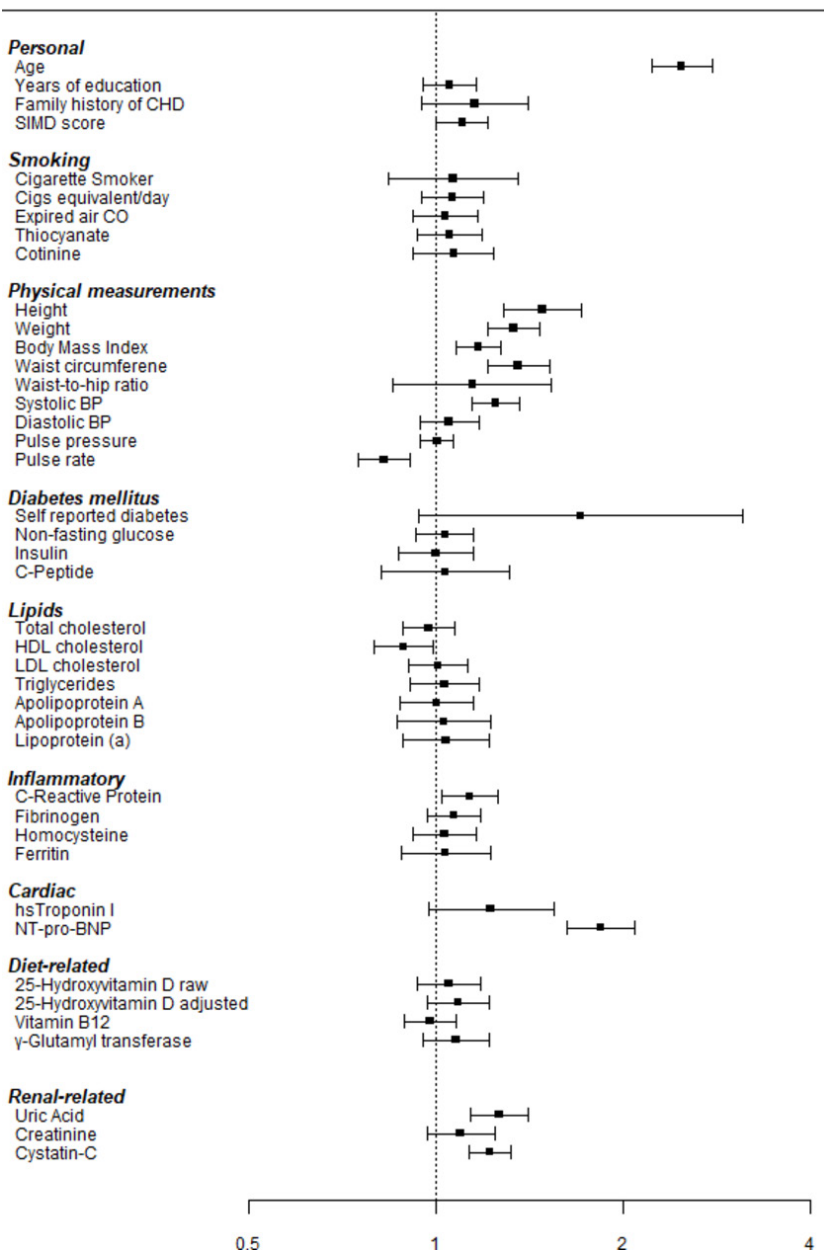

Figure 2 Multiple-adjusted $\mathrm{HRs}$, with $95 \% \mathrm{Cls}$, of the association between individual risk factors and the risk of $A F$ in women. $A F$, atrial fibrillation; $B P$, blood pressure; $C H D$, coronary heart disease; $\mathrm{CO}$, carbon monoxide; $\mathrm{HDL}$, high-density lipoprotein.

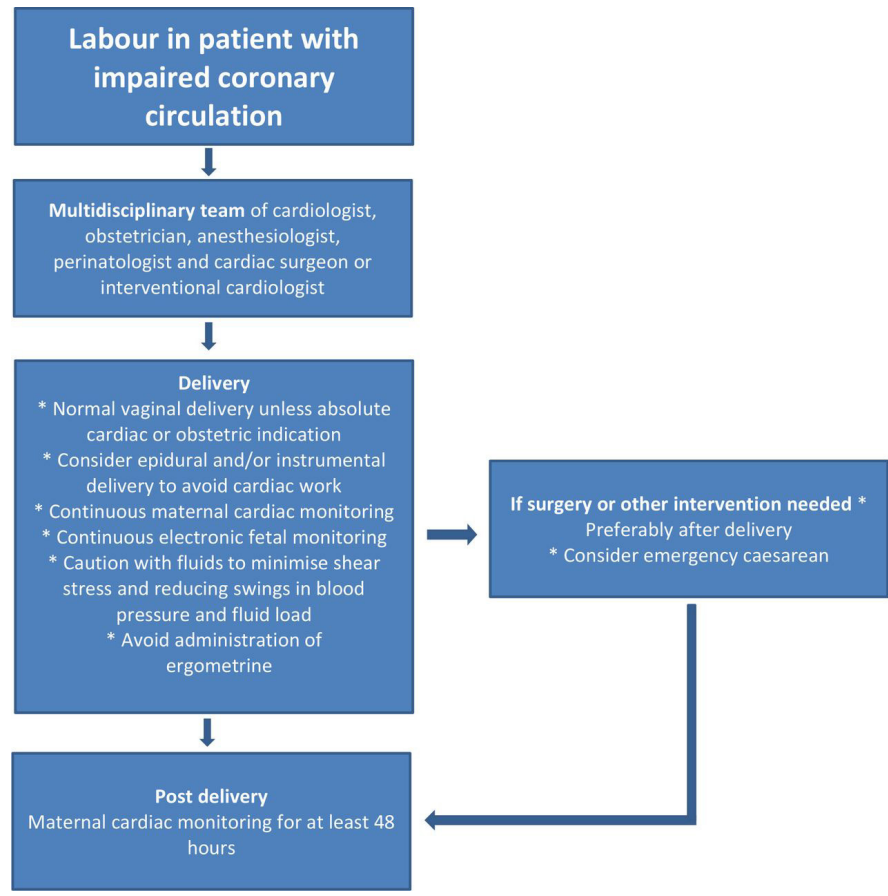

Figure 3 Delivery management for women with ischaemic heart disease. infarction. 'Thus, instead of an automatic incorporation of the current practice approach to the treatment of patients with CTO, the population coming from coronary CTA evaluation might benefit from a more selective use of revascularisation procedures.'

Atrial fibrillation (AF) is an increasing problem with our ageing population but there have been few studies on whether risk factors for $\mathrm{AF}$ are the same in women as in men. Using data on over 15 thousand participants in the Scottish Heart Health Extended Cohort, Peters and Woodward ${ }^{5}$ found that relationships were similar in men and women for risk of AF related to older age, lower socioeconomic status, taller stature, increased systolic blood pressure and higher C-reactive protein levels. However, body mass index was a stronger risk factor for $\mathrm{AF}$ in men than in women. For women, the strongest risk factors were elevated NT-pro-BNP, uric acid (a surrogate marker of oxidative stress) and cystatin-C (a marker of renal function) levels. (figure 2)

In the discussion, the authors note that these observations are important for several reasons including insights into the aetiology of $\mathrm{AF}$ that might translate into new treatment targets, more accurate projections of the future burden of $\mathrm{AF}$ allowing better allocation of healthcare resources, and improved sex-specific risk scores for identifying patients at risk of AF.

The Education in Heart article in this issue $^{6}$ reviews the current indications and contraindication to cardiac transplantation, summarises the clinical evaluation prior to listing for transplantation and discusses the long-term outcomes and complications after heart transplantation. Although the number of cardiac transplantations in the UK remains relatively low, there were over 3000 heart transplants annually in the USA in 2016 with increasing numbers each year and longer survival times after transplantation. Thus, every cardiologist should be aware of the issues involved in managing these patients and, most importantly, which patients should be referred to a specialist centre for consideration of heart transplantation.

Reader who care for pregnant women with heart disease also will want to read the review article in this issue on management of pregnant women with chronic or acute ischaemic disease (figure 3 ). ${ }^{7}$

The Image Challenge question ${ }^{8}$ (8) in this issue asks you for the most likely diagnosis in a 35 year old man with acute heart failure and these images. (figure 4) 


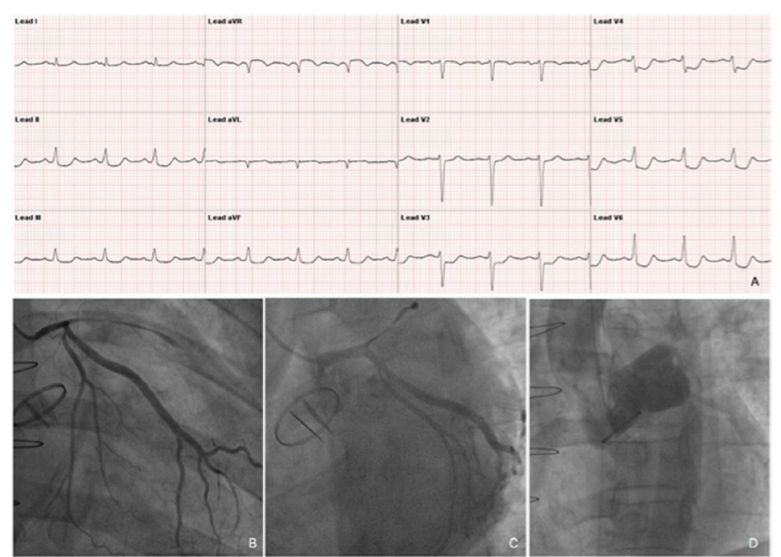

Figure 4 (A) 12-lead ECG. (B, C) Selective angiogram of the left main, left anterior descending artery and circumflex artery. (D) Aortic root angiography.

Competing interests None declared.

Patient consent for publication Not required.

Provenance and peer review Commissioned; internally peer reviewed.

(c) Author(s) (or their employer(s)) 2019. No commercial re-use. See rights and permissions. Published by BMJ.

To cite Ribeiro AL, Otto CM. Heart 2019;105:171-173.

Heart 2019;105:171-173. doi:10.1136/heartjnl-2018-314636

\section{REFERENCES}

1 Moriarty F, Bennett K, Fahey T. Fixed-dose combination antihypertensives and risk of medication errors. Heart 2019;105:204-9.

2 Webster R. Fixed-dose combination medications for non-communicable diseases. Heart 2019;105:176-7.

3 Opolski MP, Gransar H, Lu Y, et al. Prognostic value of chronic total occlusions detected on coronary computed tomographic angiography. Heart 2019;105:196-203.

4 Azevedo CF, Bittencourt MS. Can coronary computed tomography angiography be the complete roadmap for chronic total occlusion management? Heart 2019;105:174-5.

5 Peters SAE, Woodward M. Established and novel risk factors for atrial fibrillation in women compared with men. Heart 2019;105:226-34.

6 Bhagra SK, Pettit S, Parameshwar J. Cardiac transplantation: indications, eligibility and current outcomes. Heart 2019;105:251-9.

7 Cauldwell M, Baris L, Roos-Hesselink JW, et al. Ischaemic heart disease and pregnancy. Heart 2019;105:189-95.
8 Marchandot B, Radulescu B, Morel O. Chain pain following cardiac surgery in a 35-year-old man. Heart 2019;105:234. 